\title{
Integration of genomics, metagenomics, and metabolomics to identify interplay between susceptibility alleles and microbiota in adenoma initiation
}

Jacob E. Moskowitz ${ }^{1,2}$, Anthony G. Doran ${ }^{3,4}$, Zhentian Lei ${ }^{5}$, Susheel B. Busi ${ }^{1}$, Marcia L. Hart ${ }^{6}$, Craig L. Franklin ${ }^{1,6}$, Lloyd W. Sumner ${ }^{5}$, Thomas M. Keane ${ }^{3,4}$ and James M. Amos-Landgraf ${ }^{1,6^{*}}$ (i)

\begin{abstract}
Background: Colorectal cancer (CRC) is a multifactorial disease resulting from both genetic predisposition and environmental factors including the gut microbiota $(G M)$, but deciphering the influence of genetic variants, environmental variables, and interactions with the GM is exceedingly difficult. We previously observed significant differences in intestinal adenoma multiplicity between C57BL/6 J-Apc Min (B6-Min/J) from The Jackson Laboratory (JAX), and original founder strain C57BL/6JD-ApC ${ }^{\text {Min }}$ (B6-Min/D) from the University of Wisconsin.

Methods: To resolve genetic and environmental interactions and determine their contributions we utilized two genetically inbred, independently isolated $\mathrm{ApC}^{\mathrm{Min}}$ mouse colonies that have been separated for over 20 generations. Whole genome sequencing was used to identify genetic variants unique to the two substrains. To determine the influence of genetic variants and the impact of differences in the GM on phenotypic variability, we used complex microbiota targeted rederivation to generate two Apc mutant mouse colonies harboring complex GMs from two different sources (GMJAX originally from JAX or GMHSD originally from Envigo), creating four Apc ${ }^{\text {Min }}$ groups. Untargeted metabolomics were used to characterize shifts in the fecal metabolite profile based on genetic variation and differences in the GM.

\footnotetext{
* Correspondence: amoslandgrafj@missouri.edu

'Department of Veterinary Pathobiology, University of Missouri, Columbia, MO 65201, USA

${ }^{6}$ Mutant Mouse Resource and Research Center, University of Missouri, 4011 Discovery Drive, Columbia, MO 65201, USA

Full list of author information is available at the end of the article
}

(c) The Author(s). 2020 Open Access This article is licensed under a Creative Commons Attribution 4.0 International License, which permits use, sharing, adaptation, distribution and reproduction in any medium or format, as long as you give appropriate credit to the original author(s) and the source, provide a link to the Creative Commons licence, and indicate if changes were made. The images or other third party material in this article are included in the article's Creative Commons licence, unless indicated otherwise in a credit line to the material. If material is not included in the article's Creative Commons licence and your intended use is not permitted by statutory regulation or exceeds the permitted use, you will need to obtain permission directly from the copyright holder. To view a copy of this licence, visit http://creativecommons.org/licenses/by/4.0/ The Creative Commons Public Domain Dedication waiver (http://creativecommons.org/publicdomain/zero/1.0/) applies to the data made available in this article, unless otherwise stated in a credit line to the data. 
(Continued from previous page)

Results: WGS revealed several thousand high quality variants unique to the two substrains. No homozygous variants were present in coding regions, with the vast majority of variants residing in noncoding regions. Host genetic divergence between Min/J and Min/D and the complex GM additively determined differential adenoma susceptibility. Untargeted metabolomics revealed that both genetic lineage and the GM collectively determined the fecal metabolite profile, and that each differentially regulates bile acid (BA) metabolism. Metabolomics pathway analysis facilitated identification of a functionally relevant private noncoding variant associated with the bile acid transporter Fatty acid binding protein 6 (Fabp6). Expression studies demonstrated differential expression of Fabp6 between Min/J and Min/D, and the variant correlates with adenoma multiplicity in backcrossed mice.

Conclusions: We found that both genetic variation and differences in microbiota influences the quantitiative adenoma phenotype in $A p c^{\mathrm{Min}}$ mice. These findings demonstrate how the use of metabolomics datasets can aid as a functional genomic tool, and furthermore illustrate the power of a multi-omics approach to dissect complex disease susceptibility of noncoding variants.

Keywords: Genetics, Gut microbiota, Colorectal cancer, Metabolomics, Apc, Min

\section{Background}

Colorectal cancer $(\mathrm{CRC})$ is a complex disease trait resulting from a variety of factors including genetic predisposition, diet, age, inflammation, and lifestyle [1-3]. Malignant disease is preceded by the initiation of adenomas in the epithelial lining of the intestinal mucosa, and often persist up to 10 years before acquiring malignant transformations, making the adenoma a critical target for early intervention [4]. Recently, CRC has been associated with perturbations in the gut microbiota (GM) through postulated mechanisms including modulation of inflammation, genotoxin production, and metabolic homeostasis [5-8], but it is often unclear whether these shifts in bacterial composition directly impact disease risk, or merely result from physiological changes associated with disease. Initiation and progression of adenomas is likely determined by a combination of genetic factors and changes in microbial populations that mutually impact relevant pathways [9]. However, the ability to successfully integrate these complex factors and to dissect the independent and additive effects of each remains elusive in human populations.

The intestinal environment is collectively comprised of dynamic interactions between diet, modified host compounds, and microbial metabolites [10]. As such, changes in host functional genomic output via germline or acquired mutations, or shifts in the functional GM, may substantially influence the metabolite profile. Using metabolomics provides an avenue to interrogate the metabolic output of complex biological systems in a non-targeted discovery-based approach [11]. In controlled experiments, metabolites represent a highly sensitive means of detecting functional changes associated with genomic variation, differences in complex microbial communities, and even more importantly the combination of these factors in the context of complex disease traits. Several studies have demonstrated the utility of characterizing metabolite profiles in colorectal cancer, identifying microbial metabolites including short-chain fatty acids (SCFAs) such as butyrate that can influence gene expression, cell proliferation, and ultimately adenoma formation [12]. Furthermore, altered levels of microbial-influenced metabolites including bile acids (BA) and hydrogen sulfide $\left(\mathrm{H}_{2} \mathrm{~S}\right)$ are associated with both inflammatory bowel disease and CRC through the production of genotoxic reactive oxygen species $[8,13-$ 15]. As an approach, non-targeted metabolomics data correlate to $16 \mathrm{~S}$ rRNA microbiome composition more strongly than targeted metabolomics, and have identified novel metabolites in CRC patients [16].

Due to the challenges of controlling environmental conditions and performing longitudinal monitoring of disease progression from pre-disease stages in human populations, adequate models need to be refined to study early initiating events. The $A p c^{\mathrm{Min}}$ (Min) mouse model of CRC, which harbors an autosomal dominant mutation in the Apc tumor suppressor gene causing the development of intestinal adenomas, provides an extensively studied platform to interrogate genomic and GM contributions to disease initiation in a quantitative manner [17]. Investigators using this model have observed complex genetic modification of the adenoma phenotype from multiple modifier genes, including modifiers between mouse strains and newly arising variants within the C57BL/6 J strain [18-20]. It is now clear that in addition to both known and unknown genetic factors, the GM can also impact adenoma initiation and progression, as germ-free Min mice develop significantly lower adenoma burdens than their colonized counterparts [21]. Still, it is unclear how functional genomic changes and distinct GM communities independently and additively influence adenoma initiation in the context of the complex specific-pathogen-free GM. Thus, the Min mouse provides a platform to dissect genomic and 
microbial contribution to phenotypic variability, and draw further inferences about variable disease susceptibility across human populations.

A small sampling of the tumor count data reported in the Min mouse shows a wide range of small intestinal tumor counts among control animals. Throughout the course of over two decades of use of the C57BL/6 J$\mathrm{Apc}^{+/ \mathrm{Min}}$ mouse, reported adenoma counts across different colonies have varied substantially (Table 1). In some cases, these disparities were attributed to undetermined differences between institutions. It is wellestablished that mice originating from different mouse producers and institutions have highly distinct GMs [22]. Furthermore, strict genetic control of mouse models is essential to maintaining a consistent phenotype. Though producers take precautions to prevent genetic drift in inbred colonies, mutations in genetic modifiers of the Min phenotype may be selected for rapidly within a colony, and thus account for differences in tumor number across different colonies. In this study, we leveraged the observed phenotypic variability between two inbred Min colonies from a common lineage that have been separated in excess of 20 generations, to interrogate whether disparity in tumor numbers between C57BL/6 inbred colonies occurs due to differences in the GM or host genetic differences associated with colony divergence. We transferred embryos from mice from a low-tumor multiplicity colony $\left(\mathrm{C} 57 \mathrm{BL} / 6 \mathrm{~J}-\mathrm{Apc} \mathrm{C}^{\mathrm{Min}} / \mathrm{J}\right.$ abbrv. Min/J) and a high-tumor multiplicity colony (C57BL/6JMlcr- $A p c^{\text {Min }} /$ Mlcr abbrv. $\mathrm{Min} / \mathrm{D})$ into surrogate dams harboring distinct complex GMs, resulting in two genetically independent lines of mice each harboring two distinct complex GMs. We describe independent and additive influences of host genetics and the GM on adenoma initiation through the use of $16 \mathrm{~S}$ rRNA microbial profiling, host wholegenome sequencing (WGS), and finally non-targeted metabolomics. This approach allows for the relatively non-invasive identification of altered biologically

Table 1 Summary of small intestinal (SI) adenoma number variability between C57BL6/J-Apc ${ }^{\text {Min }}$ colonies

\begin{tabular}{ll}
\hline $\begin{array}{l}\text { Tumor Count } \\
\text { (II) }\end{array}$ & Reference \\
\hline 22 & $\begin{array}{l}\text { MacGregor DJ et al. International Journal of Oncology. } \\
2000 .\end{array}$ \\
34 & Zell JA et al. International Journal of Cancer. 2007. \\
41 & Chiu CH et al. Cancer Research. 1997. \\
71 & Niho N et al. Cancer Science. 2003. \\
102 & Ahn B and Ohshima H. Cancer Research. 2001. \\
108 & Paulsen JE et al. Carcinogenesis. 1997. \\
128 & Kwong et al. Genetics. 2007. \\
\hline
\end{tabular}

relevant pathways and mechanistic associations with CRC initiation through integration and refinement of large data sets.

\section{Methods}

\section{Animal use and ethics statement}

Animal studies were conducted in an Association for Assessment and Accreditation of Laboratory Animal Care International (AAALAC) accredited facility according to the guidelines provided by the Guide for the Care and Use of Laboratory Animals, and were approved by the University of Missouri Institutional Animal Care and Use Committee. For Complex Microbiota Targeted Rederivation (CMTR) C57BL/6JMlcr-Apc ${ }^{\mathrm{Min}} / \mathrm{Mmmh}$ $(\mathrm{Min} / \mathrm{D})$ and $\mathrm{C} 57 \mathrm{BL} / 6 \mathrm{~J}-A p c^{\mathrm{Min}} / \mathrm{J}(\mathrm{Min} / \mathrm{J})$ embryos were transferred into separate Crl:CD1 surrogate dams with distinct complex GM populations (GMJAX and GMHSD) to naturally deliver offspring representing four experimental groups; Min/ $/ \mathrm{J}_{\mathrm{GMJAX}}, \mathrm{Min} / \mathrm{D}_{\mathrm{GMJAX}}, \mathrm{Min} /$ $\mathrm{J}_{\mathrm{GMHSD}}$, and Min/D $\mathrm{D}_{\mathrm{GMHSD}}$ (Fig. 1a).

Male and female CMTR offspring were group-housed by sex, genetic origin of the embryo donor $(\mathrm{Min} / \mathrm{D}$ or $\mathrm{Min} / \mathrm{J}$ ), and GM of the surrogate dam (GMJAX or GMHSD). All mice, including embryo donors, ET recipients, and rederived offspring were group-housed in microisolator cages on ventilated racks (Thoren, Hazelton, PA) on a 14:10 light:dark cycle on paper chip bedding (Shepherd Specialty Papers, Watertown, TN), with ad libitum access to 5058 irradiated breeder chow (LabDiet, St. Louis, MO) and acidified autoclaved water. All pups were ear-punched at weaning (21 days of age) using sterile technique. DNA was extracted using the "HotSHOT" genomic DNA preparation method as described [23]. To generate N2 backcross animals Min/D males and WT females from the Min/J colony were first crossed to create F1 hybrids of the two genetic lineages. F1 hybrids were then backcrossed to both the Min/D and $\mathrm{Min} / \mathrm{J}$ parental lines to create $\mathrm{N} 2$ mice. At 3 months of age, all mice were euthanized via $\mathrm{CO}_{2}$ asphyxiation and the abdominal cavity was opened. Whole small and large intestines were incised longitudinally, flushed with saline and placed on bibulous paper with the luminal side facing up for formalin fixation. Grossly visible adenomas were counted manually using a Leica M165FC microscope at $1.25 \mathrm{x}$ magnification. Fecal samples were collected from all rederived mice at 1 month, while fecal samples, cecal material, and ileal scrapes were collected after sacrifice at 3 months of age.

\section{Embryo collection and transfer}

Embryos for transfer were collected from donors from two separate colonies (ET donors). Half of the embryos were obtained from frozen stocks that were generated through breeding of sexually mature C57BL/6JD- 

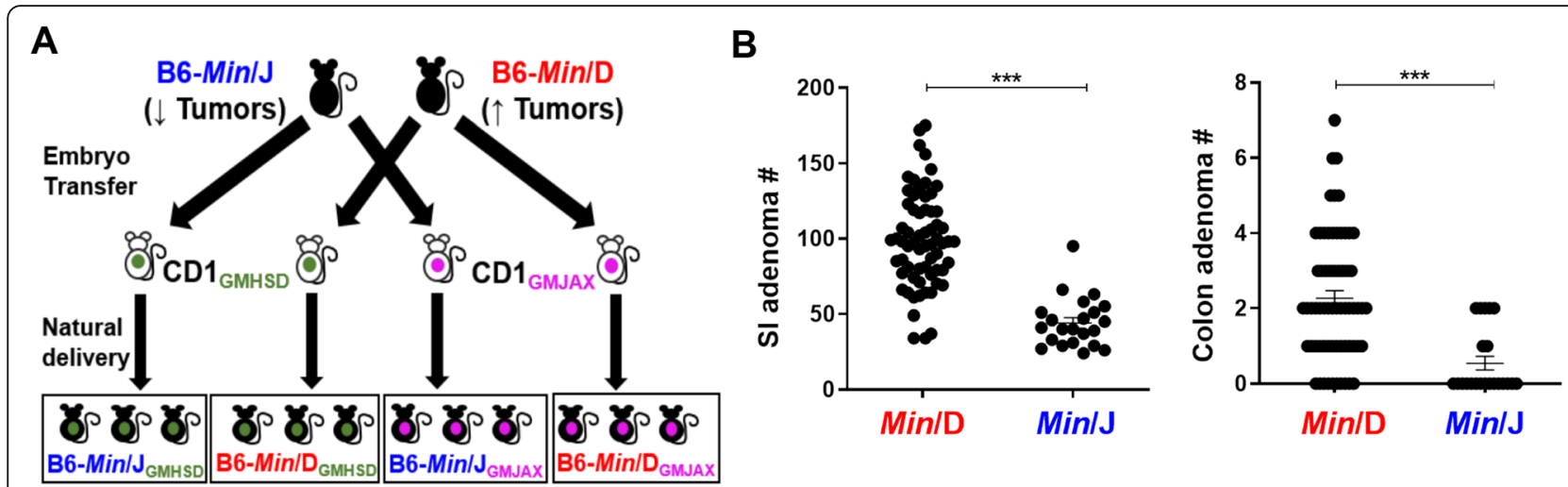

C
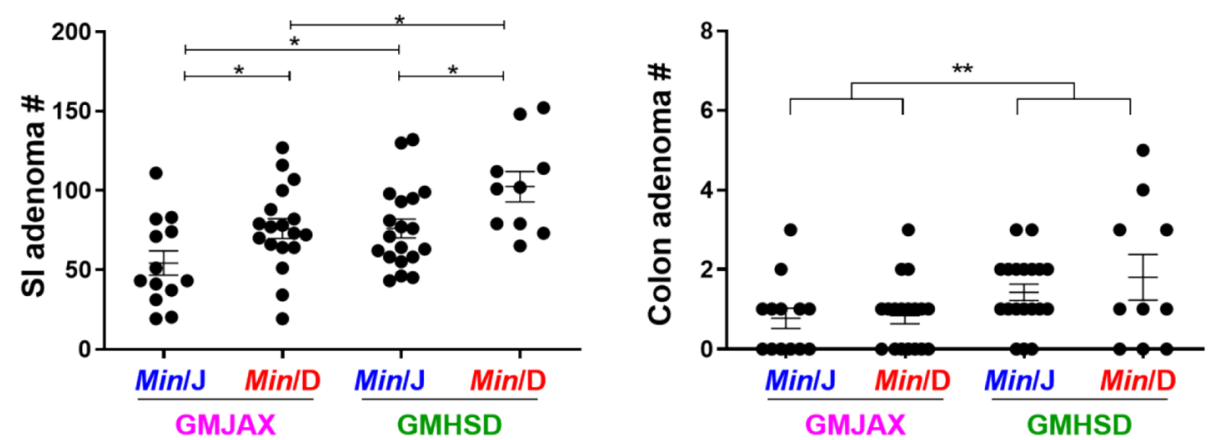

Fig. 1 Genetic lineage and GM colonization additively determine adenoma numbers in ApcMin mice. a Embryos from the Min/J and Min/D genetic lineages were transplanted into surrogate dams harboring two distinct complex GM profiles; GMJAX and GMHSD. Offspring represent the two genetic lineages which have inherited a GM from their respective surrogate dams $\left(\operatorname{Min} / J_{G M J A}, n=13 ; M i n / D_{G M J A X}, n=18 ; M i n / J_{G M H S D}, n=19\right.$; Min/D $\left.{ }_{G M H S D}, n=10\right)$. b Scatter plots comparing mean ( \pm SEM) small intestinal (SI) and colon adenoma counts of the original B6-Apc ${ }^{\text {Min }}$ colony generated at UW McArdle Laboratory (Min/D) to B6-ApC Min mice acquired from The Jackson Laboratory and maintained at University of Missouri (Min/J) (Min/D, $n=65 ;$ Min/J, $n=22)$. c Scatter plots comparing mean ( \pm SEM) SI and colon adenoma counts of the four rederived groups, including each genetic lineage (Min/J and Min/D) rederived with two complex GMs. ${ }^{*} p<0.05,{ }^{* *} p<0.01,{ }^{* * *} p<0.001$; Student's t-test (a) and Two-way ANOVA with the Student Newman-Keuls method (c)

$A p c^{+/ M i n}(\mathrm{Min} / \mathrm{D})$ males with 5-8 week-old C57BL/6JD$\mathrm{Apc}^{+/+}$females, maintained as a closed-colony at the McArdle Laboratory, University of Wisconsin (Madison, WI). A second cohort of embryos for ET was obtained on-site (University of Missouri, Columbia, MO) using C57BL/6 J-Apc ${ }^{+/ M i n}(\mathrm{Min} / \mathrm{J})$ males and 5 to 8 week-old C57BL/6 J- $A p c^{+/+}$females, purchased from The Jackson Laboratory (Bar Harbor, ME). To generate Min/J embryos, in vitro fertilization was performed as described [24]. Presumptive zygotes were then moved to a KSOM dish and incubated for $24 \mathrm{~h}$ to allow progression to the two-cell stage [25]. For ET recipients, 8 week old CD1 females harboring a GM (Hsd:CD1 $1_{\text {GMHSD }}$ ) from Envigo (Envigo, Indianapolis, IN) were purchased and allowed to acclimate for 1 week prior to use. Eight week old CD1 females harboring a GM representing The Jackson Laboratory (Crl:CD1 $1_{\mathrm{GMJAX}}$ ) were previously generated in our laboratory [26]. $\mathrm{CD} 1_{\mathrm{GMHSD}}$ and $\mathrm{CD} 1_{\mathrm{GMJAX}}$ surrogate female embryo recipients were mated with sterile, vasectomized Hsd:CD1 or Crl:CD1 males, respectively. All surrogate females were inspected for copulatory plugs and plug-positive mice were used for embryo transfer. Surrogate females were anesthetized via IM injection of ketamine/xylazine cocktail at $5.5 \mathrm{mg}$ and $1 \mathrm{mg}$ per $100 \mathrm{~g}$ body weight respectively, and placed in sternal recumbency. A dorsal midline incision was made and the uterine oviducts located by dissecting through the retroperitoneal muscle. Embryos in 3 to $5 \mu \mathrm{l}$ of media were injected into the oviducts using a glass handpipette. Skin incisions were closed with sterile surgical staples and mice received a subcutaneous injection of $2.5 \mathrm{mg} / \mathrm{kg}$ of body weight flunixin meglumine (Banamine ${ }^{\circledast}$ ) prior to recovery on a warming pad.

\section{Tissue collection and processing}

All mice were humanely euthanized with $\mathrm{CO}_{2}$ asphyxiation and necropsied, and small intestines were processed as described above. A sterile scalpel blade was used to gently scrape normal ileal epithelium. After the body cavity was opened, whole spleens and liver were 
also collected. All collected tissue was flash-frozen in liquid nitrogen followed by storage at $-80^{\circ} \mathrm{C}$.

\section{Sample collection and DNA extraction for 16S rRNA sequencing}

Two fecal pellets per mouse were collected aseptically and placed in a $2 \mathrm{~mL}$ round-bottom tube containing $800 \mu \mathrm{l}$ of lysis buffer [22] and a $0.5 \mathrm{~cm}$ diameter stainless steel bead (Grainger, Lake Forest, Il). All samples were mechanically disrupted using a TissueLyser II (Qiagen, Venlo, Netherlands) for $2 \mathrm{~min}$ at $50 \mathrm{~Hz}$, followed by incubation at $70{ }^{\circ} \mathrm{C}$ for $20 \mathrm{~min}$ with periodic vortexing. DNA extraction from fecal pellets, cecal contents, and ileal epithelium for 16S rRNA sequencing was performed using a DNeasy Blood \& Tissue Kit ${ }^{\oplus}$ (Qiagen) as previously described [22].

\section{S library preparation and sequencing}

DNA extraction from fecal pellets, cecal contents, and ileal epithelium for $16 \mathrm{~S}$ rRNA sequencing was performed using a DNeasy Blood \& Tissue Kit ${ }^{\oplus}$ (Qiagen) as previously described (See Supplemental Methods) [22]. Bacterial 16S rRNA amplicons were generated by amplification of the V4 hypervariable region of the $16 \mathrm{~S}$ rRNA gene using universal primers (U515F/806R) [27], then sequenced on the Illumina MiSeq platform as described previously [22]. Assembly, binning, and annotation of DNA sequences was performed using Qiime v1.9 [28] at the University of Missouri Informatics Research Core Facility (Columbia, MO) as described [22]. Contiguous sequences were assigned to operational taxonomic units (OTUs) using a criterion of $97 \%$ nucleotide identity by de novo clustering. Taxonomy was assigned to selected OTUs using BLAST [29] against the SILVA database [30] of $16 \mathrm{~s}$ rRNA sequences and taxonomy.

\section{Whole-genome sequencing}

Genomic DNA for whole-genome sequencing (WGS) was extracted from splenic tissue using the DNeasy Blood \& Tissue $\mathrm{Kit}^{\oplus}$, as described by the manufacturers (Qiagen). Paired-end (151 base pair) sequence reads generated for each sample were aligned to the GRCm38 (mm10) mouse reference genome using BWA-MEM (v0.7.5) [http://arxiv.org/abs/1303.3997] followed by a local realignment around indels using the GATKv3.0 'IndelRealigner Tool' [20644199]. Possible PCR and optical duplicates were filtered using Picard tools (v1.64) (http://broadinstitute.github.org/picard). SNP and short indel calls were generated using the Mouse Genomes Project variation catalog v5 parameters (described in detail [27480531]). In brief, samtools mpileup v1.3 [19505943] and bcftools call v1.3 [21653522] were used to identify SNPs and indels in each of the samples. Indels were left-aligned using the bcftools norm function. Filters were then applied to removed variants of low depth ( $<10$ reads), low genotype quality $(\mathrm{q}<20)$, poor mapping quality $(\mathrm{q}<20)$ and proximity to an indel (SNPs within $2 \mathrm{bp}$ of an indel). Additionally, only heterozygous SNPs with $>5$ support reads for each allele were retained. Functional consequences based on mouse Ensembl gene models (v88) were annotated using the Variant Effect Predictor [20562413]. The VEP tool facilitates the identification of synonymous and deleterious mutations such as stop changes and potentially damaging missense variants. Variants private to each sample were identified by removing SNPs and indels common to any of the 36 strains present in the MGPv5 catalog [27480531].

\section{TA cloning and sanger sequencing for variant validation}

As described previously in Genotyping, ear punches were used to collect DNA for variant validation. To validate the observed variant in the upstream region of Fabp6 detected by WGS, this region was PCR amplified using the primers FWD 5'-ACCACTTCCTCCCTCAGGAT-3', REV 5'TTCTCCCAATGCCCATCCAG-3'. The TOPO TA Cloning $^{\oplus}$ Kit (Invitrogen ${ }^{\text {TM }}$ ) was used to insert the region of interest into the $\mathrm{pCR}^{\mathrm{Tm}} 4-\mathrm{TOPO}^{\oplus}$ vector, and TOP10 competent $E$. coli cells were used for vector transformation according to the manufacturer's instructions. Transformed cells were spread onto Lysogeny Broth (LB) plates with 50 $\mu \mathrm{g} / \mathrm{mL}$ kanamycin for resistance selection, then grown overnight at $37^{\circ} \mathrm{C}$ in a shaking incubator. The PureYield ${ }^{\mathrm{m}}$ Plasmid Miniprep System (Promega, Madison, WI) was used to extract DNA from each culture according to the manufacturer's instructions. Sequencing reactions were prepared using the extracted DNA and the T7 sequencing primer (5'-TAATACGACTCACTATAGGG-3'. Sanger sequencing was performed at the MU DNA Core using a 3730xl 96-capillary DNA analyzer (ThermoFisher Scientific, Waltham, MA) with the Applied Biosystems Big Dye Terminator cycle sequencing chemistry.

\section{Genotyping}

Genotyping for the Min allele by PCR was performed in a reaction volume of $10 \mathrm{uL}$ containing $0.2 \mathrm{uM}$ of each primer (5'-ATTGCCCAGCTCTTCTTCCT-3' and 5'CGTCCTGGGAGGTATGAATG-3'), 1 x HRM Supermix (BioRad, Hercules, CA), and genomic DNA. Genotyping for the Fabp6 upstream insertion was similarly performed using ear-punches as described. The $10 \mathrm{uL}$ HRM reaction contained $0.2 \mathrm{uM}$ of each primer $\left(5^{\prime}\right.$ ACCACTTCCTCCCTCAGGAT-3' and 5'-TTCTCC CAATGCCCATCCAG-3'), 1 x HRM Supermix, and genomic DNA. Genotyping reactions and analyses were carried out using a BioRad CFX384 Real-Time PCR Detection system. For Min genotyping, cycling conditions were as follows: $95^{\circ} \mathrm{C}, 2 \mathrm{~min} ; 40$ cycles of $95^{\circ} \mathrm{C}, 10$ $\mathrm{s} ; \quad 60^{\circ} \mathrm{C}, \quad 30 \mathrm{~s}, \quad 72^{\circ} \mathrm{C}, \quad 30 \mathrm{~s}, 95^{\circ} \mathrm{C}, 30 \mathrm{~s} ; 60^{\circ} \mathrm{C}, 1 \mathrm{~min}$, followed by melt curve analysis from $73^{\circ} \mathrm{C}$ to $85^{\circ} \mathrm{C}$ in 
increments of $0.1{ }^{\circ} \mathrm{C}$ for $10 \mathrm{~s}$. PCR cycling conditions for Fabp 6 analysis were the same as those mentioned above, followed by a melt curve analysis from $65^{\circ} \mathrm{C}$ to $95^{\circ} \mathrm{C}$ in increments of $0.2^{\circ} \mathrm{C}$. All melt curve results were analyzed using BioRad Precision Melt Software v1.2 to detect the Min allele or the Fabp6 insertion.

\section{Tissue processing and reverse transcriptase-quantitative PCR (RT-qPCR)}

Ileal scrapes collected at 3 months of age were used to quantitate expression of Fabp6, and liver used to quantitate expression of Cyp39a1. All collected tissue was flash-frozen in liquid nitrogen followed by storage at $80^{\circ} \mathrm{C}$. Frozen tissues were mechanically disrupted using a TissueLyser II (Qiagen) for $4 \mathrm{~min}$ at $50 \mathrm{~Hz}$. Total RNA was then extracted using the AllPrep ${ }^{\circ}$ DNA/RNA Mini Kit (Qiagen), and cDNA was synthesized using the iScript $^{\mathrm{TM}}$ cDNA Synthesis Kit (Bio-Rad, Hercules, CA) according to the respective manufacturer's instructions. Samples were analyzed in quadruplicate and all evaluated gene expression levels were normalized to Hprt expression using a PrimeTime ${ }^{\oplus}$ qPCR assay $\left(\right.$ IDT $\left.^{\oplus}\right)$. For qPCR, each $10 \mathrm{uL}$ reaction contained 1 x Primer/Probe mixes (Table S9), 1 x iTaq $^{\text {Tm }}$ Universal Probe Supermix, and $100 \mathrm{ng}$ cDNA template. PCR parameters were: denaturation at $95^{\circ} \mathrm{C}$ for $5 \mathrm{~s}$, and annealing and elongation at $60^{\circ} \mathrm{C}$ for $30 \mathrm{~s}$ for a total of 54 cycles.

\section{Ultra-high performance liquid chromatography-tandem mass spectrometry (UHPLC-MS/MS)}

Fecal samples weighing $25 \mathrm{mg}$ were treated with $1.0 \mathrm{~mL}$ $80 \% \mathrm{MeOH}$ with $18 \mu \mathrm{g} / \mathrm{mL}$ umbelliferone, sonicated for $5 \mathrm{~min}$ and centrifuged for $40 \mathrm{~min}$ at $3000 \mathrm{~g}$ at $10^{\circ} \mathrm{C} .0 .5$ $\mathrm{mL}$ supernatant was used for UHPLC-MS analysis after centrifugation at $5000 \mathrm{~g}$ at $10^{\circ} \mathrm{C}$ for $20 \mathrm{~min}$ and transfer of $250 \mu \mathrm{L}$ of extract into glass vials with inserts. A Bruker maXis impact quadrupole-time-of-flight mass spectrometer coupled to a Waters ACQUITY UPLC system was used to perform UHPLC-MS analysis. Compound separation was achieved on a Waters C18 column $(2.1 \times$ $100 \mathrm{~mm}, \mathrm{BEH}$ C18 column with 1.7 -um particles) using a linear gradient and mobile phase A ( $0.1 \%$ formic acid) and B (acetonitrile). Phase B increased from 5 to $70 \%$ over $30 \mathrm{~min}$, then to $95 \%$ over $3 \mathrm{~min}$, held at $95 \%$ for 3 min, then returned to $5 \%$ for equilibrium. Flow rate was $0.56 \mathrm{~mL} / \mathrm{min}$ and the column temperature was $60{ }^{\circ} \mathrm{C}$. Mass spectrometry was performed in the negative electrospray ionization mode with the nebulization gas pressure at $43.5 \mathrm{psi}$, dry gas of $12 \mathrm{l} / \mathrm{min}$, dry temperature of $250 \mathrm{C}$ and a capillary voltage of $4000 \mathrm{~V}$. Mass spectral data were collected from 100 and $1500 \mathrm{~m} / \mathrm{z}$ and were auto-calibrated using sodium formate after data acquisition. Instrument performance was monitored by the internal standard umbelliferone and peak areas of metabolites were normalized to the internal standard. One sample from each of the four experimental groups was analyzed with automated MS/MS. Fragmentation data was compared to archived PUBCHEM and KEGG fragment databases via the MetFrag web tool (https:// msbi.ipb-halle.de/MetFragBeta/).

\section{Metabolomics data analysis}

Chromatographic data was aligned using mass and retention time with XCMS software (http://xcmsonline. scripps.edu/). Following alignment, XCMS was used to generate a relative intensity table with individual features labeled by retention time and mass for analysis in the Metaboanalyst v3.0 web program [31]. In Metaboanalyst, the interquartile range method was used to filter data. Data was normalized based on sample sums of features' relative intensity, then log transformed prior to multivariate analysis. Principle Component Analysis (PCA), putative metabolite identification, and pathway overrepresentation cloud plots were generated with XCMS, where dysregulated pathways were determined using the mummichog algorithm [32]. Metaboanalyst was used to perform hierarchical clustering using the Euclidean distance measure and Ward clustering algorithm with significantly modulated (based on ANOVA) metabolites according to experimental group, and displayed as a heat-map and dendogram. Metabolite and tumor correlation analysis was performed using small intestinal tumor counts and individual feature relative intensities across all four experimental groups, and regression graphs were generated using GraphPad Prism 8. Individually significant features were determined separately in terms of GM (compared Min/D $/ \mathrm{DMJA}_{\mathrm{A}}$ and Min/ $\mathrm{D}_{\mathrm{GMHSD}}$ ) and genetic lineage (compared of $\mathrm{Min} / \mathrm{J}_{\mathrm{GMJAX}}$ and $M i n / D_{\text {GMJAX }}$ ) by t-test in XCMS. To determine the metabolites contributing to the separation and rooting of the hierarchical clusters illustrated by the dendogram, the samples were classified into those with 'high' or 'low' colonic adenoma numbers independent of genetic lineage or GM, and a linear discriminant analysis (LDA) was performed using the LEfSe (Linear discriminant analysis Effect Size) tool on a high-computing Linux platform [33]. An LDA score of $\log _{10} 2$ or greater for any given metabolite was considered significantly differential between the high and low adenoma groups.

\section{Statistical analysis}

Statistical analyses were performed using Sigma Plot 14.0 (Systat Software Inc., Carlsbad CA). Differences in OTU relative abundance between GMJAX and GMHSD were determined using Student's t-test. To account for multiple testing, OTUs with a $p$ value $<0.001$ were considered statistically significant. Two-way ANOVA with the Student Newman-Keuls post-hoc method was used 
to assess differences in adenoma number between rederived groups, where $p<0.05$ was considered statistically significant. For GM analysis, GraphPad Prism 8 was used to generate bar graphs and Tukey's box plots displaying phylum relative abundances, richness (OTU counts), and $\alpha$-diversity (Shannon Index). Principal Coordinate Analyses incorporating the Bray-Curtis similarity index used for visualizing $\beta$-diversity were generated with the Paleontological Statistics software package (PAST) 3.12 [34]. Two-way ANOVA/Student Newman-Keuls posthoc method was used to assess differences in richness and $\alpha$-diversity and phylum abundance differences between rederived mice. To better account for quantitative and qualitative community differences between GMJAX and GMHSD, statistical testing for $\beta$-diversity was performed via a two-way PERMANOVA analysis of both Bray-Curtis and Jaccard dissimilarities for bacterial OTU community structure using PAST 3.12. For RT-qPCR assays, expression analysis was performed using the $2^{-\Delta \Delta C t}$ method of relative expression [35], and statistical differences were assessed using the Student's t-test.

\section{Results}

Genetic lineage and GM colonization additively determine adenoma susceptibility in distinct C57BL/6-Apc ${ }^{\mathrm{Min}}$ colonies

Historically, tumor multiplicities in C57BL/6-Apc ${ }^{\text {Min }}$ mice vary widely in reported studies despite having the same inbred genetic background (Table 1). Notably, these colonies were housed in different institutions for unknown numbers of generations prior to reporting tumor numbers, highlighting the difficulty in separating the impact of genetic divergence from environmental variables. We compared intestinal adenoma number in our institution between two C57BL/6- $A p c^{\mathrm{Min}}$ lines arising from a common colony. The original B6-Apc ${ }^{\mathrm{Min}}$ colony was developed in the McArdle Laboratory at the University of Wisconsin $(M i n / D)$. A subset of $M i n / D$ mice were sent to the Jackson Laboratory (JAX) and underwent rederivation for colony development and distribution $(\mathrm{Min} / \mathrm{J})$, and thus harbor a GM representing JAX. The original Min/D colony was maintained as a closed colony through sibling mating and harbored a GM from Harlan/Sprague Dawley (now Envigo) that was acquired through pup fostering to ICR (Hsd:ICR (CD1)) foster mice to rid the colony of Helicobacter spp. Mice from the Min/D colony had an average of 99.2 small intestinal (SI) and 2.26 colonic adenomas [36], and breeder males were consistently progeny-tested to maintain tumor multiplicities in the offspring within one standard deviation from the average. The Min/J colony acquired from the Jackson Laboratory and maintained at the University of Missouri had significantly fewer SI and colonic adenomas, with 44.2 and 0.55 , respectively (SI and colon $p<0.001$ ) (Fig. 1a).

To interrogate how GM and host genetic lineage independently and additively contribute to variable adenoma susceptibility in $A p c^{M i n}$ mice, we used Complex Microbiota Targeted Rederivation (CMTR) to establish mice from the $\mathrm{Min} / \mathrm{J}$ genetic lineage and the Min/D genetic lineage with two different complex GMs; a low-richness GM originally acquired from B6 mice from the Jackson Laboratory (GMJAX) and high-richness GM originally acquired from CD-1 mice from Envigo (GMHSD). These GM profiles were chosen because they most closely represent the original GMs of the $\mathrm{Min} / \mathrm{J}$ and $\mathrm{Min} / \mathrm{D}$ colonies, respectively. $\mathrm{Min} / \mathrm{J}$ and $\mathrm{Min} / \mathrm{D}$ embryos were separately implanted into surrogate dams harboring the desired GM, such that they would maintain their original genetic lineage while acquiring the desired maternal GM through natural birth. Thus, we generated four experimental groups representing each combination of genetic lineage and GM (Fig. 1b). All $A p c^{M i n}$ offspring were sacrificed at 3 months of age, and SI and colonic adenomas were counted to determine the effects of genetic lineage and GM colonization on adenoma susceptibility. We found that independent of genetic lineage, mice colonized with GMHSD developed more SI adenomas than their GMJAX counterparts. Furthermore, when comparing adenoma susceptibility between the genetic lineages within each GM, mice of the Min/D lineage developed more adenomas than Min/J mice independent of GM (Fig. 1c). Thus, colonization of Min/J embryos with GMHSD partially restored the original Min/D phenotype, but did not account entirely for the phenotypic differences between the original Min/D and $\mathrm{Min} / \mathrm{J}$ colonies. Colonization of $\mathrm{Min} / \mathrm{D}$ embryos with GMJAX suppressed the original Min/D phenotype, while colonization of $M i n / D$ with GMHSD completely restored the original McArdle phenotype. Combining the effects of genetic lineage and GM, Min/D $\mathrm{D}_{\mathrm{GMHSD}}$ mice develop substantially more adenomas than $\mathrm{Min} / \mathrm{J}_{\mathrm{GMJAX}}$ $(p<0.001)$. In the colon, we observed increased adenomas in GMHSD-colonized mice compared to GMJAX, while genetic lineage had no apparent effect (Fig. 1c). These trends were similarly observed when males and females were assessed separately (Fig. S1). To summarize, both genetic lineage and GM colonization independently modulated adenoma susceptibility, and collectively had either additive protective or deleterious phenotypic effects.

\section{Distinct GM communities influence adenoma susceptibility}

To characterize the GMJAX and GMHSD microbial communities, feces were collected at 1 month, and fecal 
and ileal epithelial scrapes at 3 months of age, from rederived $A p c^{M i n}$ mice. $16 \mathrm{~S}$ rRNA sequencing was then used to determine relative abundance of all detected microbial taxa. At 1 month, phyla Proteobacteria, Actinobacteria, Deferribacteres, and Cyanobacteria were enriched in GMHSD-colonized mice, while Tenericutes were enriched in GMJAX-colonized mice (Fig. 2a). These changes were observed regardless of genetic lineage, indicating that phylum make-up was determined by the surrogate dam rather than genetic lineage of the embryo. At the operational taxonomic unit (OTU) level, GMJAX and GMHSD had distinct post-weaning microbial profiles in fecal samples (Fig. 2b) which remained disparate until sacrifice at 3 months in both feces and ileal scrapes (Fig. S2A). Community analyses of fecal and ileal $\beta$-diversity by two-way PERMANOVA corroborated the discrete nature of these communities (Table S1 and S2). Sex did not appear to play a significant role in GM make-up, and as anticipated based on previous characterization of these GMs [22], GMHSD mice had increased microbial richness (Chao1 index) and $\alpha$ diversity (Shannon Index) compared to GMJAX mice (Figs. S2B and S2C). Using a $p$-value of 0.001 as a threshold, we found 58 and 34 significantly modulated OTUs in feces and ileal scrapes, respectively, between GMJAX and GMHSD (Tables S3 and S4). GMHSD mice harbored enriched abundances of sulfidogenic Desulfovibrio and Bilophila sp., as well as sulfatase-secreting bacteria (SSB) Rikenella, while GMJAX had enriched levels of Bacteroides sp. and family Peptococcaceae. A heat map illustrating fold difference in the relative abundance of the 25 most significantly different OTUs was used for a hierarchical clustering analysis, and shows that samples clustered based on GM profile, regardless of genetic lineage (Fig. 2c). Thus, GMJAX and GMHSD represent highly distinct complex microbial communities with a number of different taxa potentially contributing to differential adenoma susceptibility.

\section{GM and host genetic lineage shape the metabolome in $A p C^{\mathrm{Min}}$ mice}

Based on the results of our rederivation experiment, we aimed to determine functional differences between each genetic lineage and GM community that could contribute to differential disease susceptibility using a metabolomics approach. Feces contains not only microbial metabolites, but also mammalian host metabolites that may undergo microbial biotransformation [10]. In an untargeted analysis of fecal metabolites at 3 months of age detected by liquid chromatography coupled mass spectrometry (LC-MS), we observed distinct metabolite profiles based on both genetic lineage and GM colonization (Fig. 3a). Using a False Discovery Rate (qvalue) of 0.1 as a threshold, we found that 1009 features were significantly modulated between the four rederived $A p c^{M i n}$ groups. Of these features, 172 were specifically modulated by the GM and 7 by genetic lineage (Supplementary datasets 1-3; Figs. S3A and B), while the remainder appear to be modulated by a combination of the two factors. A heat map illustrating fold-change of the most substantially modulated metabolites (based on ANOVA) was used for a hierarchical clustering analysis. This analysis demonstrated that samples primarily clustered based on GM, with a secondary clustering pattern based on genetic lineage (Fig. 3b). Notably, we found that certain metabolites had significant positive and negative correlations with adenoma number across all four rederived groups (Fig. 3c). A pathway analysis using putative compounds was performed to determine metabolic pathways modulated based on genetic lineage and GM colonization. Differential bile acid metabolism was observed when comparing $\mathrm{Min} / \mathrm{J}$ and $\mathrm{Min} / \mathrm{D}$ genetics, as defined by enrichment of putative bile acid intermediates

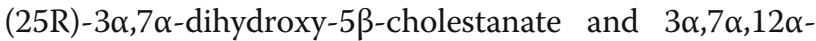
trihydroxy-24-oxo- $5 \beta$-cholestanoyl CoA in Min/D mice compared to Min/J (Table S5, Fig. 3d). Meanwhile, differential sphingosine lipid metabolism was observed based on GM colonization (Table S5). To summarize, a minority of differential features were specifically modulated by GM colonization or host genetic lineage, whereas the vast majority of features were modulated by a combination of the two factors. Furthermore, both individual metabolites and metabolic pathways were independently modulated based on genetic lineage or GM.

\section{Host genetic lineage influences bile acid metabolism}

The divergent genetic lineages $M i n / J$ and $M i n / D$ had significantly altered adenoma susceptibility and metabolic profile. We therefore characterized genetic divergence between the $\mathrm{Min} / \mathrm{J}$ and $\mathrm{Min} / \mathrm{D}$ lines via $~ 30 \mathrm{X}$ whole-genome sequencing (WGS) on representative breeder female mice from each colony (see supplementary data and methods). SNPs and indels that were private to either $\mathrm{Min} / \mathrm{D}$ or $\mathrm{Min} / \mathrm{J}$ were categorized based on their predicted functional effect due to the nature of the variant using the Variant Effect Predictor (VEP) tool (Table S6). There were no private protein coding homozygous variants detected in either line, with all homozygous variants residing in noncoding regions. To interrogate overall effects of private mutations in each lineage, all private homozygous variants residing within or near known genes were used to identify overrepresented KEGG [37] and REACTOME [38] biological pathways using the over-representation tool in InnateDB, which revealed over-representation of bile-acid metabolism in the Min/D line (Table S8) [39]. Variants near or within candidate genes contributing to bile acid metabolism included Cyp39a1, which codes for an 
A
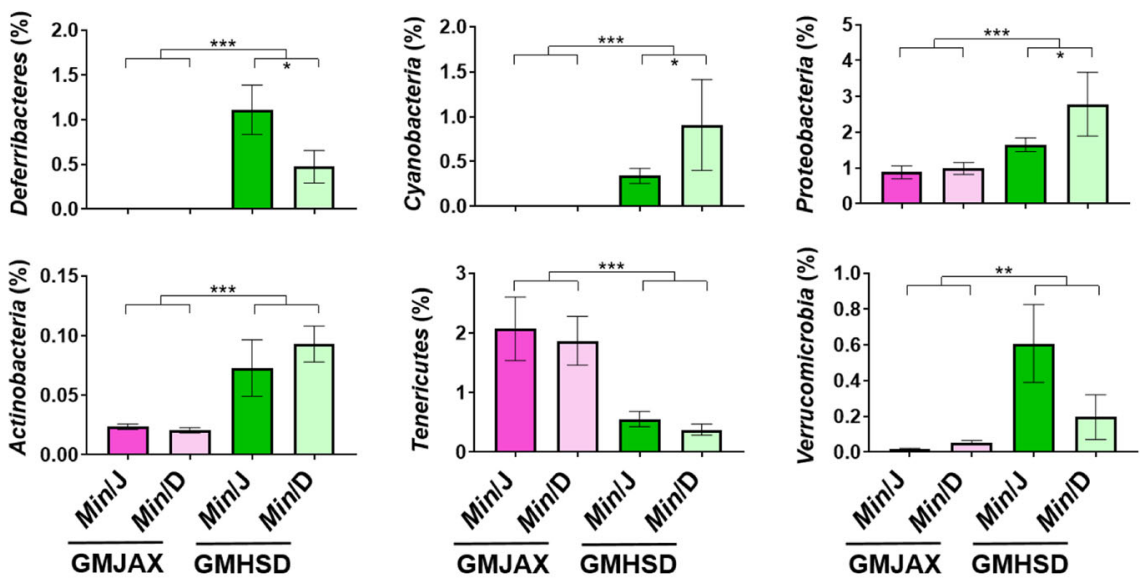

B
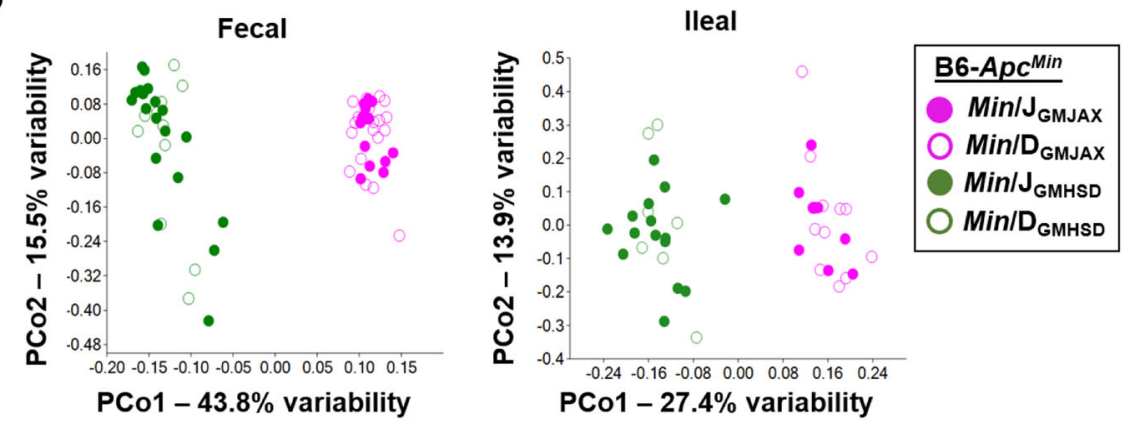

C

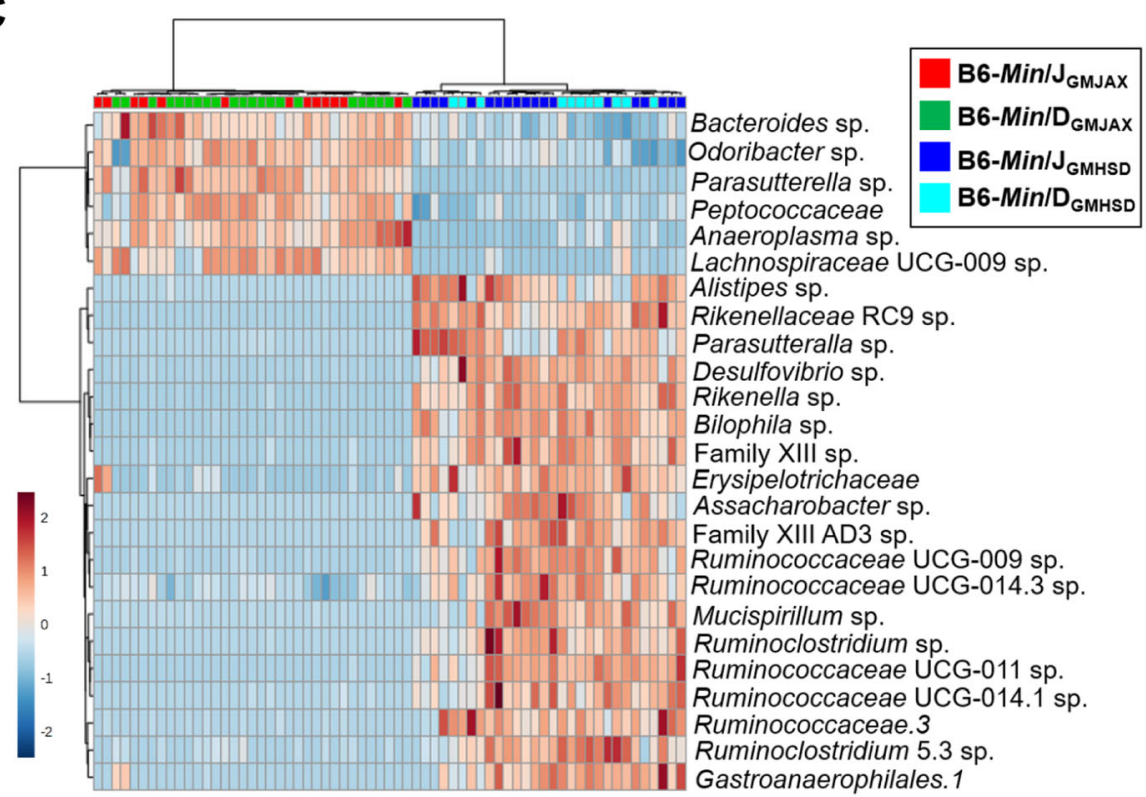

Fig. 2 (See legend on next page.) 
(See figure on previous page.)

Fig. 2 Distinct GM communities influence adenoma susceptibility. a Bar charts representing relative abundances (mean \pm SEM) of Phyla with detected significant differences between fecal samples GMJAX and GMHSD groups (Min/J JMJAX, $n=13 ; \operatorname{Min} / \mathrm{D}_{\mathrm{GMJAX}}, n=18 ; \operatorname{Min} / \mathrm{J}_{\mathrm{GMHSD}}, n=19 ; \operatorname{Min} / \mathrm{D}_{\mathrm{GMHSD}}, n=10$ ). b Unweighted Principal Coordinate Analysis (PCOA) representing differences in $\beta$-diversity at the Operational Taxanomic Unit (OTU) level between complex GM profiles of CMTR offspring in feces at 1 month, and ileal scrapes at 3 months of age. $\mathbf{c}$ Heatmap showing 25 taxa with significantly different $(p<0.001)$ fecal relative abundances between GMJAX and GMHSD at 1 month, where color intensity represents fold-change of each OTU. Hierarchical clustering based on Euclidean distances (top) demonstrates clustering of samples based on GM. All statistically significant OTUs and associated log-fold changes are represented in supplementary Tables $3 \mathrm{~A}$ (fecal) and $3 \mathrm{~B}$ (ileal). ${ }^{*} p<0.05,{ }^{* *} p<0.01,{ }^{* *} p<0.001$; Two-way ANOVA with the Student Newman-Keuls method for Multiple Comparisons

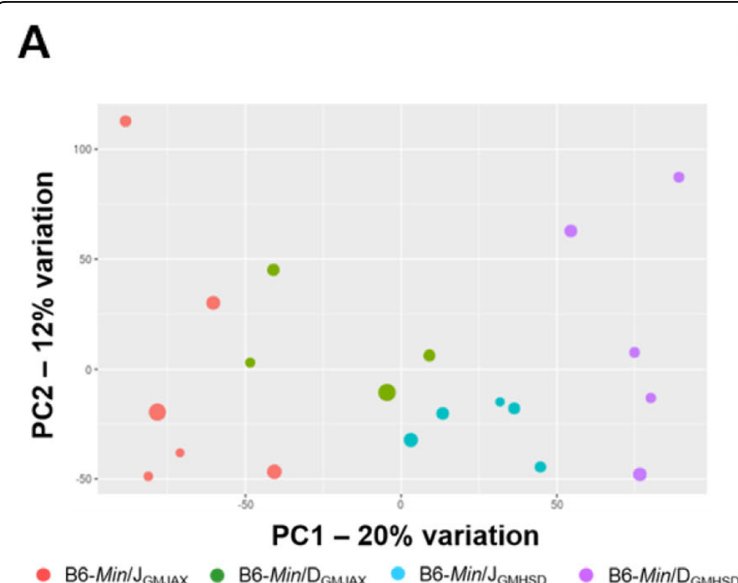

B
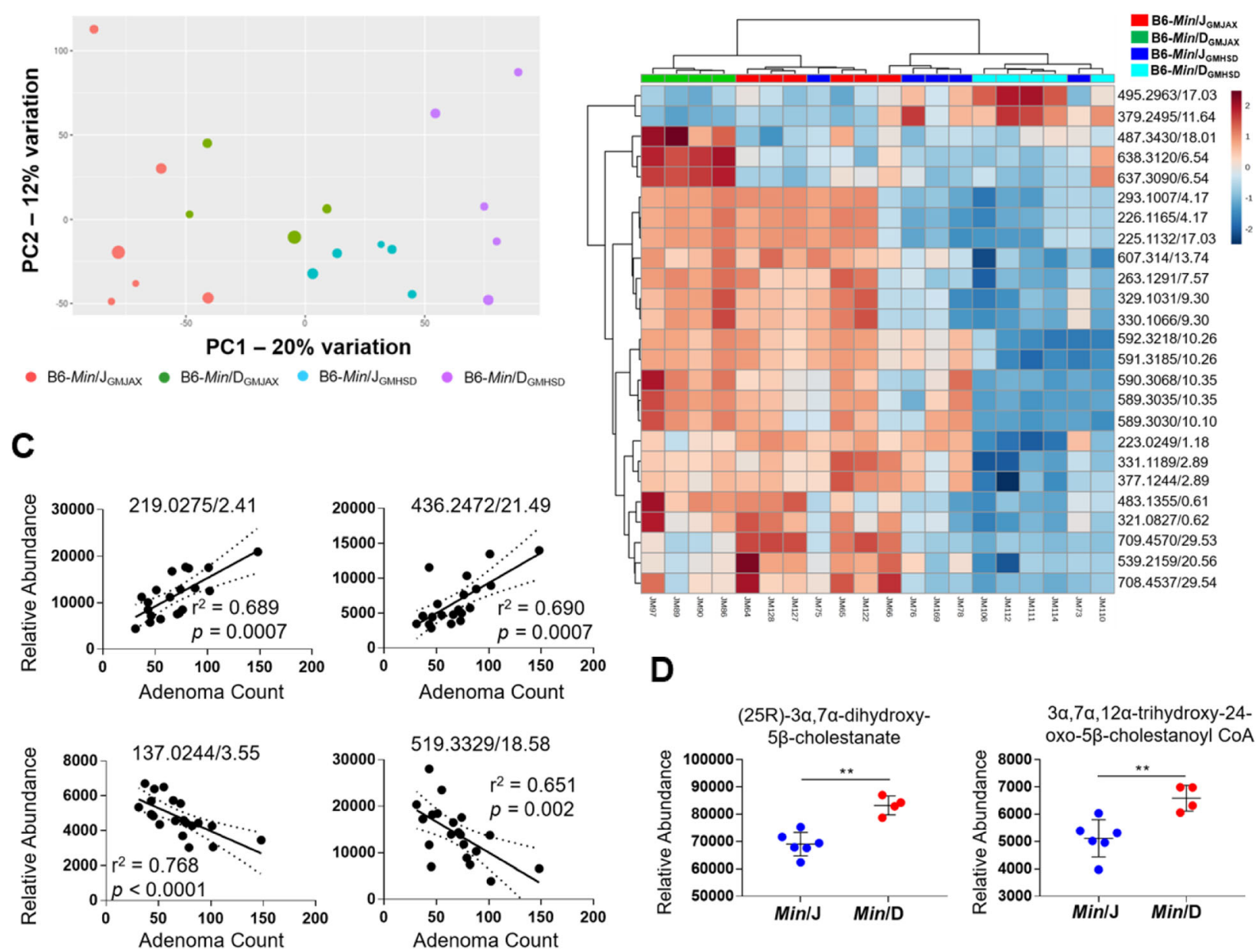

Fig. 3 Untargeted analysis of GM and host genetic lineage effects on the fecal metabolome. a PCA illustrating unsupervised clustering of fecal metabolites at 3 months of age $\left(\operatorname{Min} / J_{\text {GMAX }}, n=6 ; \operatorname{Min} / \mathrm{D}_{\mathrm{GMJAX}}, n=4 ; \operatorname{Min} / \mathrm{J}_{\mathrm{GMHSD}}, n=5 ; \operatorname{Min} / \mathrm{D}_{\mathrm{GMHSD}}, n=5\right)$. b Heatmap showing 25 detected fecal metabolites with most significantly different relative abundances across all rederived groups, where color intensity represents log-fold-change of each metabolite. Hierarchical clustering based on Euclidean distances (top) illustrates primary clustering of samples based on GM, and secondary clustering based on genetic lineage. All metabolites shown on heat map have significantly different mean abundances $(p<0.001)$ based on ANOVA. c Spearman's rank correlation was used to show metabolites with significant positive or negative correlations to SI tumor number across all rederived Apc ${ }^{\text {Min }}$ groups $(n=20)$. $\mathbf{d}$ Scatter plots of mean \pm SEM relative abundances of putative metabolites contributing to modulation of bile acid metabolism (Min/J, $n=6 ;$ Min/D, $n=4)$. Metabolites are denoted by mass:charge ratio and retention time $\left(\mathrm{m} / \mathrm{z}_{-} \mathrm{t}_{\mathrm{R}}\right){ }^{*} p<0.05,{ }^{* *} p<0.01,{ }^{* * *} p<0.001$; Student's t-test 
enzyme involved in bile acid biosynthesis, and the intestinal bile acid transporter coded for by Fabp6 [40, 41]. The Min/D line carried a single base (A) deletion in intron 1 of Cyp39a1 at position chr17:43,674,583. Min/D also carried a $\mathrm{T}_{6}$ bp insertion within a poly $\mathrm{T}$ in the area upstream of Fabp6 within the area of chr11:43,604,913$43,604,928$. Notably, homozygous variants private to the $\mathrm{Min} / \mathrm{J}$ line were detected near candidate genes $M y c$ and Dlg3 among other cancer related genes (Table S7).

\section{The Min/D Fabp6 variant is associated with SI adenoma susceptibility}

Our WGS findings of variants associated with bile acid metabolism were particularly notable as they provide a possible explanation for the previously described changes in bile acid metabolites (Fig. 3d). However, it is unclear whether there are any functional consequences of the observed germline mutations. To determine whether detected Fabp6 and Cyp39a1 variants had potential downstream effects in the tissues they are normally expressed, we compared gene expression levels in the normal ileal epithelium and liver, respectively, of $\mathrm{Min} / \mathrm{J}$ and Min/D mice. We found that Min/D mice had a significant reduction in Fabp6 expression in the ileal epithelium compared to $\mathrm{Min} / \mathrm{J}$ mice, while there were no differences in Cyp39a1 mRNA levels in the liver (Fig. 4b).

We validated the Fabp6 variant detected by WGS and further interrogated whether Fabp6 is a possible modifier of adenoma susceptibility. The $\mathrm{Min} / \mathrm{D}$ and $\mathrm{Min} / \mathrm{J}$ parental lines were used to generate N2 mice. Tumor number assessment that was performed blinded for genotype showed a significant correlation with the Fabp6 variants, where mice that were homozygous for the Min/D variant had the highest adenoma susceptibility. Those that were heterozygous displayed an intermediate phenotype, while mice that were homozygous for the Min/J variant had the lowest adenoma multiplicity (Fig. 4b). Thus, we observed differential Fabp6 expression between the $\mathrm{Min} / \mathrm{J}$ and $\mathrm{Min} / \mathrm{D}$ lineages associated with the validated upstream insertion in Min/ $\mathrm{D}$ mice, and further associated this variant with SI adenoma susceptibility in N2 mice.

\section{Colonic adenoma susceptibility is associated with changes in bile acid metabolism}

We finally aimed to determine whether the fecal metabolome could account for the observed differences in colonic adenoma number between the original Min/D and Min/J colonies (Fig. 1a). An unsupervised dendrogram was generated to cluster the fecal metabolomes from 3 month old mice based on detected putative fecal metabolite features. The major root of the tree clustered samples into two distinct groups independent of genetic lineage and GM profile (Fig. 5a). Analysis of these two groups revealed that they correlated with colonic adenoma multiplicity, indicated by the numbers adjacent to the dendrogram, where one group had $0.75 \pm 0.22$ colon adenomas, while the other had $2.5 \pm 0.57$ colon adenomas. Linear Discriminant Analysis (LDA) was used to identify the metabolites driving separation between the low-adenoma and high-adenoma clusters. In total, we found 16 metabolites associated with the high-adenoma cluster, and 6 metabolites associated with the lowadenoma cluster (Fig. 5b). Of these metabolites, tandem MS enabled identification of two metabolites overrepresented in the low-adenoma cluster, both of which were bile acid or bile acid derivatives. The relative abundance of putative cholate was primarily modulated by GM, while the abundance of putative $3 \beta, 7 \alpha, 12 \alpha$-Trihydroxy- $5 \alpha$-cholan-24-oic acid was dependent on both GM and genetic lineage (Fig. 5c). In conclusion, an unbiased clustering analysis of the fecal metabolomes of the rederived $A p c^{M i n}$ groups generated two primary
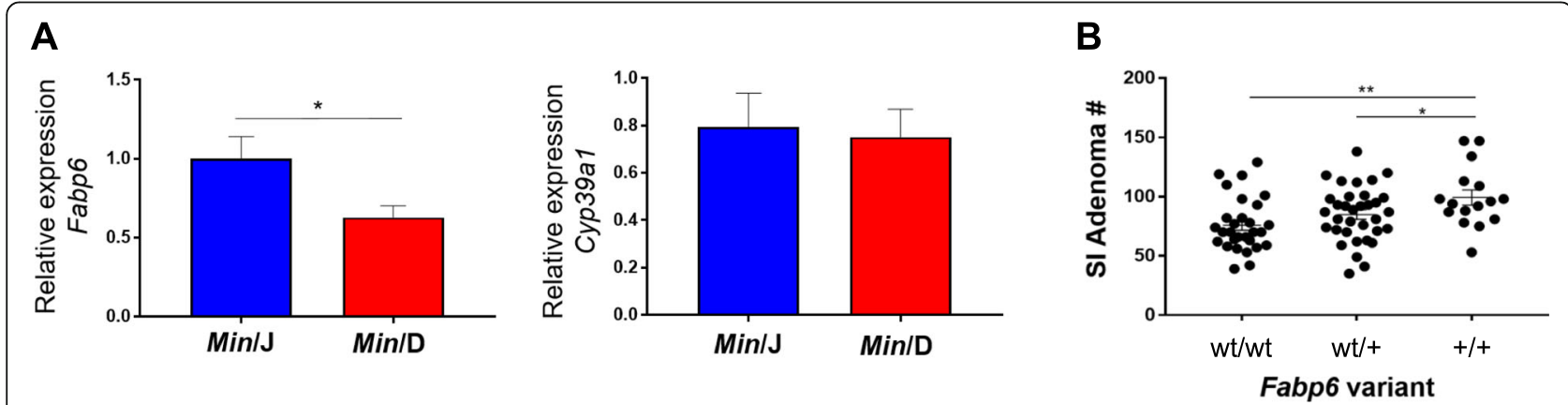

Fig. $4 \mathrm{Min} / \mathrm{D}$ Fabp6 variant association with SI adenoma susceptibility. a RT-qPCR comparison of relative expression of candidate genes Fabp6 and Cyp39a1 between Min/J and Min/D lineages, using ileal mucosal scrapes from normal intestinal epithelium and liver, respectively (Min/J, $n=10 ;$ Min/D, $n=12$ ). $\mathbf{b}$ Scatter plots comparing mean $( \pm$ SEM) SI tumor counts of N2 mice based on their status for the Fabp6 insertion (wt/wt homozygous for absence of insertion, $n=29$; wt/+ heterozygous for insertion, $n=34)+/+$ homozygous for presence of the Min/D insertion, $n=16)$. ${ }^{*} p<0.05$, ${ }^{* *} p<$ $0.01,{ }^{* * *} p<0.001$; Student's t-test (a) and ANOVA with the Student Newman-Keuls method (b) 


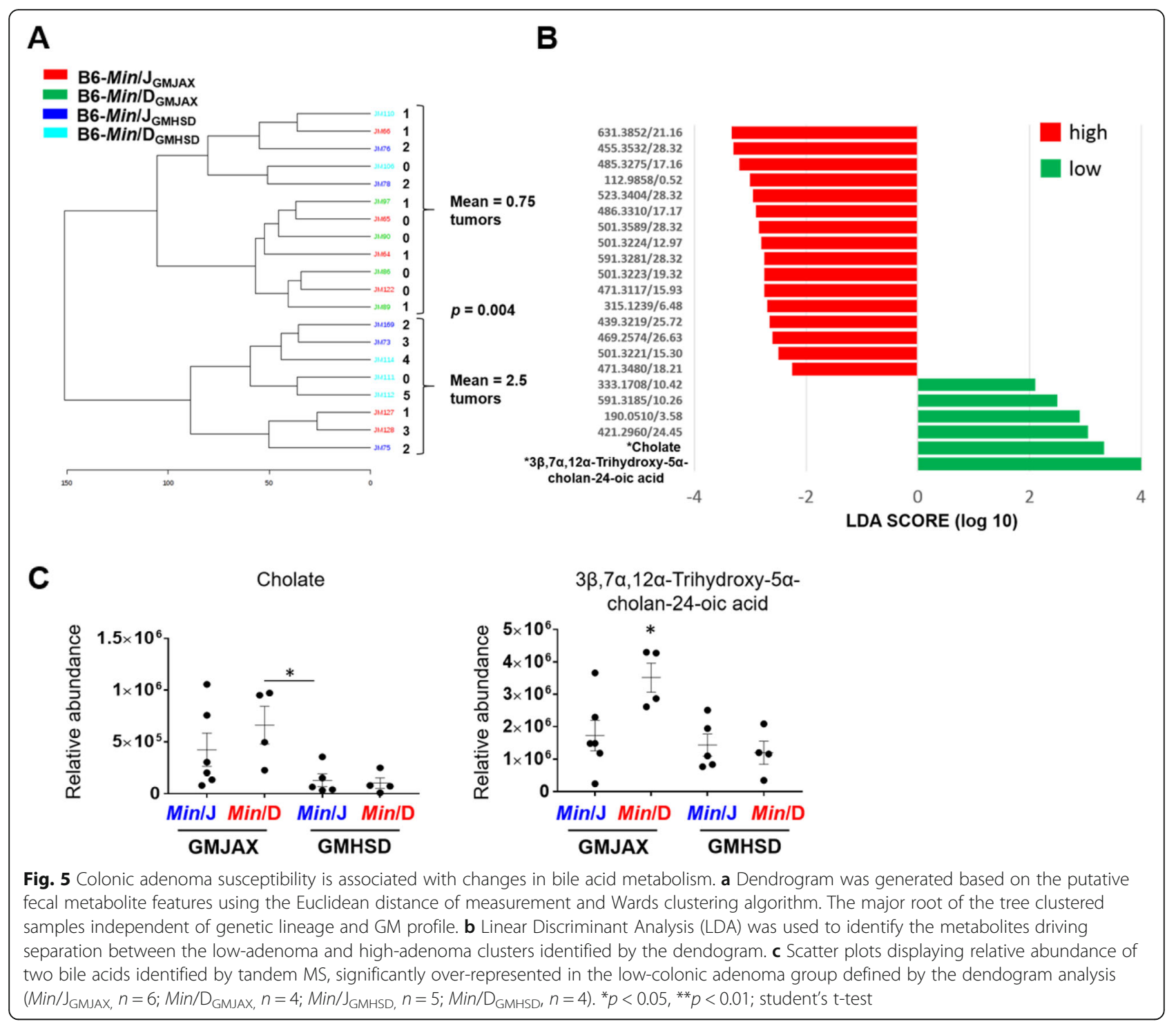

groups, which were associated with colonic adenoma numbers. Identification of two of these metabolites driving the low- and high-adenoma clusters revealed elevated levels of two bile acid compounds in the lowadenoma group, while the remainder are currently uncharacterized.

\section{Discussion}

The Min mouse is the single most cited mouse model of human cancer for nearly three decades, yielding an extraordinary wealth of information about the pathogenesis and treatment of human disease. However, the use of the Min mouse model for quantitative analysis of tumor susceptibility and treatment has been confounded by phenotypic variability of unknown origin, particularly with respect to adenoma multiplicity (Table 1). Here, we demonstrate how leveraging the observed phenotypic variability between Min colonies allows us to unravel the complex factors comprising disease susceptibility, with special focus on how host genetics and the gut microbiota (GM) collectively influence adenoma initiation. We utilized a multi-omics approach to integrate microbial community and host genomic data, and include the fecal metabolome to incorporate these data sets to provide new insight into the functional contributions of these interactions in CRC susceptibility.

We exploited our observation of a variable phenotype between two colonies that diverged from a common population; the original $\mathrm{C} 57 \mathrm{BL} / 6-A p c^{\text {Min }}$ colony generated and housed at the McCardle Laboratory at the University of Wisconsin $(\mathrm{Min} / \mathrm{D})$ and mice received from The Jackson Laboratory $(\mathrm{Min} / \mathrm{J})$ (Fig. 1a). Given the multigeneration segregation of the two colonies and the differences in selective pressures, we hypothesized that host 
genetic divergence would account for differences in adenoma susceptibility, despite having the same original inbred genetic background. Previous studies have also demonstrated that mice housed in different institutions have distinct GMs due to environmental differences, so we further hypothesized that the Min/D and Min/J colonies' disparate GM communities could contribute to distinct phenotypes [22]. The original Min/D colony was rederived onto Hsd:CD1 surrogate dams from Envigo (previously Harlan) at the McCardle Laboratory, and therefore had a GM representing Envigo, while $\mathrm{Min} / \mathrm{J}$ mice have a relatively less complex GM from The Jackson Laboratory [26]. To segregate the effects of host genetics and GM on adenoma susceptibility, we used a unique targeted rederivation approach in which we generated mice of the $M i n / D$ and $\mathrm{Min} / \mathrm{J}$ genetic lineages each with a GM representing either Envigo (GMHSD) or The Jackson Laboratory (GMJAX) (Fig. 1b). Remarkably, we demonstrated that both genetic lineage and GM considerably influenced adenoma numbers. While $\mathrm{Min} / \mathrm{J}$ mice colonized with GMHSD had increased adenoma numbers compared to our original Min/ J colony, colonization of Min/D mice with GMJAX repressed adenoma numbers compared to the original Min/ D colony, emphasizing a critical role for the GM in disease susceptibility in $A p c^{\text {Min }}$ mice (Fig. 1c). Furthermore, rederived mice of the Min/D lineage developed more adenomas than their Min/J counterparts independent of GM colonization, indicating that genetic lineage similarly accounts for significant phenotypic variability (Fig. 1c). Thus, we demonstrate here that host genetics and the GM collectively accounted for the adenoma number disparity between two divergent colonies, additively determining adenoma multiplicity.

Microbial profiling via NGS of the $16 \mathrm{~S}$ rRNA gene allows characterization of the GMJAX and GMHSD communities to identify OTUs associated with a protective versus deleterious phenotype. Analysis of $\beta$-diversity of the microbial taxa of GMHSD and GMJAX in the ileum and feces across multiple time points confirmed that these GMs are stably distinct from one another throughout the GI tract (Figs. 2 and S2). Desulfovibrio sp. and Bilophila sp., deltaproteobacteria producers of hydrogen sulfide $\left(\mathrm{H}_{2} \mathrm{~S}\right)$ via sulfate and sulfite reduction, respectively, were 2-3 orders of magnitude higher in GMHSD compared to GMJAX in both ileal scrapes and feces (Tables S3 and S4) suggesting a potentially important role for sulfidogenic bacteria. A number of studies describe associations between $\mathrm{H}_{2} \mathrm{~S}$ and $\mathrm{CRC}$ risk, indicating both pro- and anticarcinogenic roles depending on concentration and route of cellular exposure [13, 42-44]. Of further interest, Bilophila sp., named for their close association with bile, is the only bacterial genera known to utilize taurine from taurine-conjugated bile acids for anaerobic respiration and $\mathrm{H}_{2} \mathrm{~S}$ production $[8,45]$. Due to its use of bile acids as a source of respiration, $B$. wadsworthia expands dramatically in western diets with higher fat content associated with increased taurine-conjugated bile acids, and thus presents a critical link between western diets, bile acid levels, sulfide production, and CRC risk [46]. While these suggestive results remain correlative, experiments focused on supplementing these bacteria in an environmentally controlled setting could provide additional insight into complex community structures and their role in CRC pathogenesis.

The emergence of targeted and untargeted metabolomics provide an avenue to interrogate metabolic changes in disease. While the high sensitivity of an untargeted approach yields large numbers of metabolites of interest, distinguishing these compounds from unclassified fragments or adducts poses a significant challenge [16]. This study in particular exemplifies the challenges of an untargeted approach, as the vast majority of detected differential metabolite features remain unidentified. It is also important to consider that more extensive annotation of certain metabolite classes may cause inherent bias when interpreting results. Thus, continued efforts to improve metabolite libraries, as well as bioinformatics pipelines that enable more efficient compound identification, are critical to the development of these approaches.

Despite these challenges, a wealth of information can be gleaned from controlled metabolomics studies. These data show that both the GM and host genetics shape the fecal metabolome, and in the process, could alter predisposition to adenoma initiation (Fig. 2a-b). Additional analysis enables mapping of differential putative compounds to metabolic pathways, and thus shows the perturbation of such metabolic pathways associated with pathology of interest. We identified dysregulation of bile acid metabolism in mice from the $\mathrm{Min} / \mathrm{D}$ genetic lineage. The enterohepatic BA system is a classic example of the inter-dependent nature of host genetics and the GM. Host gene expression of enzymes responsible for primary BA biosynthesis, as well as intestinal transporters that recycle these BAs are required for functional enterohepatic circulation [47], while the GM de-conjugates and transforms primary bile acids as they traverse the GI tract to produce secondary BAs [48]. Thus, intra-intestinal levels of BAs depend upon cooperative genomic and microbial function.

Gleaning functional genomic significance of WGS variants is often especially challenging due to high numbers of misreads and unknown intergenic effects of poorly annotated functional elements. Thus, we used our metabolomics data, specifically identification of bile acid dysregulation, as a functional genomic tool to focus our variants of interest. We identified an insertion at a Spi1 transcription factor binding site of Fatty acid binding protein 6 (Fabp6), a protein responsible for the re- 
uptake of bile acids in the ileum for enterohepatic recirculation [49], in Min/D mice. This variant was associated with decreased expression of Fabp6 in the Min/D population, suggesting a functional role for the insertion. Previous studies have implicated Fabp6 in human CRC where it was over-expressed in cancerous tissue relative to normal tissue. Counterintuitively, higher expression levels of Fabp6 within tumors correlated with smaller tumors and less metastasis, suggesting its potential role in early carcinogenesis [41]. Decreased expression of Fabp6 in normal ileal epithelium associated with increased adenomagenesis in Min/D mice, as well as a significant association between the Fabp6 variant and SI adenoma multiplicity in N2 mice, support the proposed role for Fabp6 in tumor initiation (Fig. 4b).

While it is difficult to discern contributing factors to colonic adenoma development in $A p c^{\mathrm{Min}}$ mice due to low colon tumor numbers and an incompletely penetrant phenotype, metabolomics may provide a foundation for identifying changes associated with a more severe or suppressed colonic phenotype. An unbiased analysis of fecal metabolites in our rederived groups separated the metabolic profiles into two distinct groups defined by colonic tumor number. Among several associated compounds, we identified two bile acids where increased abundance was associated with the lowadenoma group (Fig. 5). Previous studies have implicated secondary bile acids such as deoxycholic acid (DCA) in CRC pathogenesis through mechanisms including oxidative damage and mitochondrial dysfunction, while primary bile acids can inhibit adenoma formation [50-52]. Cholic acid (CA) is a primary bile acid converted to DCA by the gut microbiota [53]. Enrichment of cholate in the low-adenoma group, and its association with GMJAX-colonized mice, may indicate that GMJAX converts CA to DCA less efficiently than GMHSD, and therefore confers a suppressed adenoma phenotype. These results highlight the diversity of bile acids and their potential effects on host cell proliferation in CRC, and suggest that carcinogenesis may depend upon a delicate balance between the two. However, further targeted studies are required to better characterize dysregulation of primary and secondary bile acids, and to determine how genetic variants and the microbiota each influence these metabolites.

\section{Conclusions}

Colorectal cancer is a classic example of a multifaceted disease with complex biological systems contributing to overall susceptibility and pathogenesis. Here, we demonstrate that complex GM communities and host genetics both independently and additively modulate adenoma development in $A p c^{M i n}$ mice. We utilized a metabolomics platform to show that genetically divergent host genomes and complex GM interactively shape the intestinal metabolome. Our strategy of utilizing untargeted metabolomics data as a functional genomics tool enabled us to focus our attention to WGS variants of consequence. Thus, we demonstrate a tactic to extract pathologically relevant functional candidate variants from large sequencing data sets. This work provides a platform for both mechanistic links between genetic variants and the GM as well as biomarker discovery. Furthermore, this data provides a clear explanation for much of the variability observed in the $A p c^{M i n}$ tumor phenotype throughout its use over the course of several decades, and may explain substantial differences in susceptibility to CRC across different human populations. Finally, this approach was relatively non-invasive and can be translated to human studies, integrating the complicated interactive nature of the host genome, the GM, and the metabolome to create individualized risk assessment and tailored preventive medicine strategies.

\section{Supplementary information}

Supplementary information accompanies this paper at https://doi.org/10. 1186/s12885-020-07007-9.

\section{Additional file 1. \\ Additional file 2. \\ Additional file 3. \\ Additional file 4.}

\section{Abbreviations}

CRC: colorectal cancer; GM: gut microbiota; Apc: Adenomatous polyposis coli; Min: Multiple intestinal neoplasia allele; Min/J: C57BL/6 J-ApC ${ }^{\text {Min }}$ mice; Min/D: C57BL/6JD-Apc ${ }^{\text {Min }}$ mice; Fabp6: Fatty acid binding protein 6; BA: bile acids; $\mathrm{H}_{2} \mathrm{~S}$ : hydrogen sulfide; GMJAX: complex gut microbiota with original origins from the Jackson Laboratory; GMHSD: complex gut microbiota with original origins from Harlan Sprague Dawley (Envigo)

\section{Acknowledgments}

We would like to thank William Dove for generously donating the C57BL/6JMlcr$\mathrm{ApC}^{\text {Min }}$ /Mmmh mice to the MMRRC for public distribution (MMRRC:043849-MU/ GMJAX and MMRRC:050543-MU/GMHSD) and comments on the manuscript. We would also like to acknowledge the contributions of Nathan Bivens and the MU DNA Core for assistance with $16 \mathrm{~S}$ rRNA gene sequencing, Bill Spollen and the MU Informatics Research Core Facility for assistance with processing and analysis of 165 rRNA sequencing data, MU Office of Animal Resources and their staff for assistance with animal husbandry and veterinary care.

\section{Authors' contributions}

All authors have read and approved this manuscript. JM and JAL collectively conceived the experimental design for all described experiments and were responsible for overseeing all mouse studies, data analysis, interpretation, and writing the manuscript. SB processed mouse tissue as required for subsequent assays including $16 \mathrm{~S}$ rRNA sequencing, metabolomics, and expression analyses. TK oversaw whole-genome sequencing, and AD was responsible for the computational processing of resulting WGS data. CF conceived the development of distinct standardized complex microbiota communities to be used as surrogates for rederivations in the described study, and $\mathrm{MH}$ implemented and maintained the surrogate populations required for this experiment. LWS oversaw all described metabolomics studies, and ZL was responsible for performing LC-MS experiments and assisted with metabolomics data processing. 


\section{Funding}

This research was funded by grants from the National Institutes of Health to the Mutant Mouse Resource and Research Center at the University of Missouri (U42 OD010918), and by the University of Missouri to Dr. James Amos-Landgraf (Startup-funding). JEM was supported by NIH T32 OD011126.

\section{Availability of data and materials}

The datasets in this publication have been made available for public access. Microbiome sequence data is available in the NCBI Sequence Read Archive. SRA: SRP216253 BioProject: PRJNA555614. The 16S sample metadata is listed in the supplementary data. The metabolomics metadata is listed in supplemental and data is available through the public database metabolomics workbench (amoslandgrafj_20200522_151621_mwtab).

\section{Ethics approval and consent to participate}

Animal studies were conducted in an Association for Assessment and Accreditation of Laboratory Animal Care International (AAALAC) accredited facility according to the guidelines provided by the Guide for the Care and Use of Laboratory Animals, and were approved by the University of Missouri Institutional Animal Care and Use Committee.

\section{Consent for publication}

All the authors consent to publication, the data has been made public as indicated in the manuscript.

\section{Competing interests}

The authors declare no competing interests.

\section{Author details}

'Department of Veterinary Pathobiology, University of Missouri, Columbia MO 65201, USA. ${ }^{2}$ Present Address: F. Widjaja Foundation Inflammatory Bowel and Immunobiology Research Institute, Cedars-Sinai Medical Center, Los Angeles, CA 90048, USA. ${ }^{3}$ Wellcome Trust Sanger Institute, Wellcome Genome Campus, Hinxton CB10 1SA, UK. ${ }^{4}$ European Bioinformatics Institute Wellcome Genome Campus, Hinxton CB10 1SD, UK. ${ }^{5}$ Department of Biochemistry, MU Metabolomics Center, University of Missouri Bond Life Sciences Center, Columbia, MO 65201, USA. ${ }^{6}$ Mutant Mouse Resource and Research Center, University of Missouri, 4011 Discovery Drive, Columbia, MO 65201, USA

\section{Received: 4 February 2020 Accepted: 26 May 2020}

Published online: 29 June 2020

\section{References}

1. Thune I, Lund E. Physical activity and the risk of colorectal cancer in men and women. Br J Cancer. 1996:73:1134-40.

2. de la Chapelle A. Genetic predisposition to colorectal cancer. Nat Rev Cancer. 2004:4(10):769-80.

3. Itzkowitz SH, Yio X. Inflammatation and Cancer IV. Colorectal cancer in inflammatory bowel disease: the role of inflammation. Am J Physiol Gastrointest Liver Physiol. 2004;287:G7-G17.

4. Bertagnolli MM, Hsu M, Hawk ET, Eagle CJ, Zauber AG. Adenoma prevention with Celecoxib study I: statin use and colorectal adenoma risk: results from the adenoma prevention with celecoxib trial. Cancer Prev Res (Phila). 2010; 3(5):588-96.

5. Couturier-Maillard A, Secher T, Rehman A, Normand S, De Arcangelis A, Haesler R, Huot L, Grandjean T, Bressenot A, Delanoye-Crespin A, et al. NOD2-mediated dysbiosis predisposes mice to transmissible colitis and colorectal cancer. J Clin Invest. 2013:123(2):700-11.

6. Cuevas-Ramos G, Petit CR, Marcq I, Boury M, Oswald E, Nougayrede JP. Escherichia coli induces DNA damage in vivo and triggers genomic instability in mammalian cells. Proc Natl Acad Sci U S A. 2010;107(25): 11537-42.

7. Travaglione S, Fabbri A, Fiorentini C. The Rho-activating CNF1 toxin from pathogenic E. coli: a risk factor for human cancer development? Infect Agent Cancer. 2008;3:4

8. Carbonero F, Benefiel AC, Alizadeh-Ghamsari AH, Gaskins HR. Microbial pathways in colonic sulfur metabolism and links with health and disease. Front Physiol. 2012;3:1-11.

9. Birt DF, Phillips GJ. Diet, genes, and microbes: complexities of colon cance prevention. Toxicol Pathol. 2014;42(1):182-8.
10. Franzosa EA, Sirota-Madi A, Avila-Pacheco J, Fornelos N, Haiser HJ, Reinker S, Vatanen T, Hall AB, Mallick H, Mclver LJ, et al. Gut microbiome structure and metabolic activity in inflammatory bowel disease. Nat Microbiol. 2019;4(2): 293-305.

11. Bino RJ, Hall RD, Fiehn O, Kopka J, Saito K, Draper J, Nikolau BJ, Mendes P, Roessner-Tunali $\mathrm{U}$, Beale $\mathrm{MH}$, et al. Potential of metabolomics as a functional genomics tool. Trends Plant Sci. 2004;9(9):418-25.

12. Bultman SJ, Jobin C. Microbial-derived butyrate: an oncometabolite or tumor-suppressive metabolite? Cell Host Microbe. 2014;16(2):143-5.

13. Attene-Ramos MS, Wagner ED, Gaskins HR, Plewa MJ. Hydrogen sulfide induces direct radical-associated DNA damage. Mol Cancer Res. 2007;5(5): 455-9.

14. Dossa AY, Escobar O, Golden J, Frey MR, Ford HR, Gayer CP. Bile acids regulate intestinal cell proliferation by modulating EGFR and FXR signaling. Am J Physiol Gastrointest Liver Physiol. 2016:310(2):G81-92.

15. Farhana L, Nangia-Makker P, Arbit E, Shango K, Sarkar S, Mahmud H, Hadden T, Yu Y, Majumdar AP. Bile acid: a potential inducer of colon cancer stem cells. Stem Cell Res Ther. 2016;7(1):181.

16. Melnik AV, da Silva RR, Hyde ER, Aksenov AA, Vargas F, Bouslimani A, Protsyuk I, Jarmusch AK, Tripathi A, Alexandrov T, et al. Coupling targeted and untargeted mass spectrometry for Metabolome-microbiome-wide association studies of human fecal samples. Anal Chem. 2017;89(14):7549-59.

17. Shoemaker AR, Gould KA, Luongo C, Moser AR, Dove WF. Studies of neoplasia in the min mouse. Biochim Biophys Acta. 1997;1332:F25-48.

18. Cormier RT, Bilger A, Lillich AJ, Halberg RB, Hong KH, Gould KA, Borenstein N, Lander ES, Dove WF. The Mom ${ }^{\text {AKR }}$ intestinal tumor resistance region consists of Pla2g2a and a locus distal to D4Mit64. Oncogene. 2000;19:3182-92.

19. Baran AA, Silverman KA, Zeskand J, Koratkar R, Palmer A, McCullen K, Curran WJ Jr, Edmonston TB, Siracusa LD, Buchberg AM. The modifier of min 2 (Mom2) locus: embryonic lethality of a mutation in the Atp5a1 gene suggests a novel mechanism of polyp suppression. Genome Res. 2007;17(5): $566-76$

20. Kwong LN, Dove WF. APC and its modifiers in colon cancer. Adv Exp Med Biol. 2009:656:85-106.

21. Li Y, Kundu P, Seow SW, de Matos CT, Aronsson L, Chin KC, Karre K, Pettersson S, Greicius G. Gut microbiota accelerate tumor growth via c-Jun and STAT3 phosphorylation in APCMin/+ mice. Carcinogenesis. 2012;33(6):1231-8.

22. Ericsson AC, Davis JW, Spollen W, Bivens N, Givan S, Hagan CE, McIntosh M, Franklin CL. Effects of vendor and genetic background on the composition of the fecal microbiota of inbred mice. PLoS One. 2015;10(2):e0116704.

23. Truett GE, Heeger P, Mynatt RL, Truett AA, Walker JA, Warman ML. Preparation of PCR-quality mouse genomic DNA with hot sodium hydroxide and tris (HotSHOT). BioTechniques. 2000;29(1):52 54.

24. Takeo TNN. Reduced glutathione enhances fertility of frozen/thawed C57BL/ 6 mouse sperm after exposure to methyl-Beta-Cyclodextrin. Biol Reprod. 2011:85:1066-72.

25. Biggers JDML, Raffin M. Amino acids and Preimplantation development of the mouse in protein-free potassium simplex optimized medium. Biol Reprod. 2000;63:281-93.

26. Hart ML, Ericsson AC, Lloyd KCK, Grimsrud KN, Rogala AR, Godfrey VL, Nielsen JN, Franklin CL. Development of outbred CD1 mouse colonies with distinct standardized gut microbiota profiles for use in complex microbiota targeted studies. Sci Rep. 2018;8(1):10107.

27. Caporaso JG, Lauber CL, Walters WA, Berg-Lyons D, Lozupone CA, Turnbaugh PJ, Fierer N, Knight R. Global patterns of $16 \mathrm{~S}$ rRNA diversity at a depth of millions of sequences per sample. Proc Natl Acad Sci U S A. 2010;108:4516-22.

28. Kuczynski J, Stombaugh J, Walters WA, Gonzalez A, Caporaso JG, Knight R: Using QIIME to analyze 16S rRNA gene sequences from microbial communities. Curr Protoc Microbiol 2012, Chapter 1:Unit 1 E 5.

29. Altschul SF, Madden TL, Schaffer AA, Zhang J, Zhang Z, Miller W, Lipman DJ. Gapped BLAST and PSI-BLAST: a new generation of protein database search programs. Nucleic Acids Res. 1997;25(17):3389-402

30. Ritari J, Salojarvi J, Lahti L, de Vos WM. Improved taxonomic assignment of human intestinal $16 \mathrm{~S}$ rRNA sequences by a dedicated reference database. BMC Genomics. 2015:16:1056.

31. Xia J, Sinelnikov IV, Han B, Wishart DS. MetaboAnalyst 3.0--making metabolomics more meaningful. Nucleic Acids Res. 2015:43(W1):W251-7.

32. Smith CA, Want EJ, O'Maille G, Abagyan R, Siuzdak G. XCMS: processing mass spectrometry data for metabolite profiling using nonlinear peak alignment, matching, and identification. Anal Chem. 2006;78(3):779-87. 
33. Segata N, Izard J, Waldron L, Gevers D, Miropolsky L, Garrett WS, Huttenhower C. Metagenomic biomarker discovery and explanation. Genome Biol. 2011;12(6):R60.

34. Hammer $\varnothing$, Harper DAT, Ryan PD. Past: paleontological statistics software package for education and data analysis. Palaeontologia Electronica. 2001; 1(4):9.

35. Schmittgen TD, Livak KJ. Analyzing real-time PCR data by the comparative CT method. Nat Protoc. 2008;3(6):1101-8.

36. Amos-Landgraf JMHJ, Wielenga MCB, Dunkin E, Krentz KJ, et al. Sex disparity in colonic adenomagenesis involves promotion by male hormones, not protection by female hormones. PNAS. 2014;111(46):16514-9.

37. Kanehisa M, Goto S. KEGG: Kyoto Encylcopedia of genes and genomes. Nucleic Acids Res. 2000;28(1):27-30

38. Joshi-Tope G, Gillespie M, Vastrik I, D'Eustachio P, Schmidt E, de Bono B, Jassal B, Gopinath GR, Wu GR, Matthews L, et al. Reactome: a knowledgebase of biological pathways. Nucleic Acids Res. 2005;33(Database issue):D428-32.

39. Breuer K, Foroushani AK, Laird MR, Chen C, Sribnaia A, Lo R, Winsor GL, Hancock RE, Brinkman FS, Lynn DJ. InnateDB: systems biology of innate immunity and beyond--recent updates and continuing curation. Nucleic Acids Res. 2013;41(Database issue):D1228-33.

40. Fuchs M. Bile Acid Regulation of Hepatic Physiology III. Rregulation of bile acid synthesis: past progress and future challenges. Am J Physiol Gastrointest Liver Physiol. 2003;284:G551-9.

41. Ohmachi T, Inoue H, Mimori K, Tanaka F, Sasaki A, Kanda T, Fujii H, Yanaga K, Mori M. Fatty acid binding protein 6 is overexpressed in colorectal cancer. Clin Cancer Res. 2006;12(17):5090-5.

42. Attene-Ramos MS, Wagner ED, Plewa MJ, Gaskins HR. Evidence that hydrogen sulfide is a genotoxic agent. Mol Cancer Res. 2006;4(1):9-14.

43. Hellmich MR, Szabo C. Hydrogen sulfide and Cancer. Handb Exp Pharmacol. 2015;230:233-41.

44. Guo FF, Yu TC, Hong J, Fang JY. Emerging roles of hydrogen sulfide in inflammatory and neoplastic colonic diseases. Front Physiol. 2016;7:156.

45. Laue H, Friedrich M, Ruff J, Cook AM. Dissimilatory sulfite reductase (desulfoviridin) of the taurine-degrading, non-sulfate-reducing bacterium Bilophila wadsworthia RZATAU contains a fused DsrB-DsrD subunit. J Bacteriol. 2001:183(5):1727-33.

46. Ridlon JM, Kang DJ, Hylemon PB, Bajaj JS. Bile acids and the gut microbiome. Curr Opin Gastroenterol. 2014;30(3):332-8.

47. Thomas C, Pellicciari R, Pruzanski M, Auwerx J, Schoonjans K. Targeting bile-acid signalling for metabolic diseases. Nat Rev Drug Discov. 2008;7(8):678-93.

48. Ridlon JM, Kang DJ, Hylemon PB. Bile salt biotransformations by human intestinal bacteria. J Lipid Res. 2006;47(2):241-59.

49. Lefebvre P, Cariou B, Lien F, Kuipers F, Staels B. Role of bile acids and bile acid receptors in metabolic regulation. Physiol Rev. 2009;89(1):147-91.

50. Cao H, Luo S, Xu M, Zhang Y, Song S, Wang S, Kong X, He N, Cao X, Yan F, et al. The secondary bile acid, deoxycholate accelerates intestinal adenomaadenocarcinoma sequence in Apc ( $\mathrm{min} /+)$ mice through enhancing Wnt signaling. Familial Cancer. 2014;13(4):563-71.

51. Degirolamo C, Modica S, Palasciano G, Moschetta A. Bile acids and colon cancer: solving the puzzle with nuclear receptors. Trends Mol Med. 2011; 17(10):564-72

52. Smith DL, Keshavan P, Avissar U, Ahmed K, Zucker SD. Sodium taurocholate inhibits intestinal adenoma formation in APCMin/+ mice, potentially through activation of the farnesoid X receptor. Carcinogenesis. 2010;31(6): $1100-9$

53. Brown DG, Rao S, Weir TL, O'Malia J, Bazan M, Brown RJ, Ryan EP. Metabolomics and metabolic pathway networks from human colorectal cancers, adjacent mucosa, and stool. Cancer Metab. 2016;4:11.

\section{Publisher's Note}

Springer Nature remains neutral with regard to jurisdictional claims in published maps and institutional affiliations.

Ready to submit your research? Choose BMC and benefit from:

- fast, convenient online submission

- thorough peer review by experienced researchers in your field

- rapid publication on acceptance

- support for research data, including large and complex data types

- gold Open Access which fosters wider collaboration and increased citations

- maximum visibility for your research: over $100 \mathrm{M}$ website views per year

At $\mathrm{BMC}$, research is always in progress.

Learn more biomedcentral.com/submissions 